\title{
Boundary conditions for Grad's 13 moment equations
}

\author{
Toby Thatcher, Y. Zheng, and H. Struchtrup* \\ Department of Mechanical Engineering \\ University of Victoria, Victoria BC, Canada \\ *Corresponding author: struchtr@uvic.ca
}

\begin{abstract}
A complete set of boundary conditions for Grad's 13 moment equations is derived from Maxwell's boundary conditions for the Boltzmann equation. The equations are solved for plane Couette flow. The results exhibit temperature jump and slip, and agree well with DSMC calculations for Knudsen numbers $\mathrm{Kn} \leq 0.1$. Nonlinear effects lead to unphysical results at larger Knudsen numbers, and for very fast flows. A simplified version of the Grad 13 equations, the so-called bulk equations, gives meaningful results in conditions where the full set of equations fails.
\end{abstract}

Keywords: Kinetic Theory, microscale flows, Grad moment method, boundary conditions

Reference to this paper should be made as follows: Thatcher, T., Zheng, Y., and Struchtrup, H. (2006) 'Boundary conditions for Grad's 13 moment equations', Progress in Computational Fluid Dynamics, Vol. X, pp.xx-yy.

Biographical notes: Henning Struchtrup is Associate Professor at the Department of Mechanical Engineering at the University of Victoria, Victoria BC, Canada. He completed his $\mathrm{PhD}$ degree from the Technical University Berlin (Germany) in 1996. His research lies in Kinetic theory of gases, non-equilibrium thermodynamics, and transport theory.

\section{Introduction}

Flow processes in rarefied gases, that is at Knudsen numbers above 0.02 (say), cannot be described by the classical Navier-Stokes and Fourier laws, since rarefaction effects occur. Rarefied flows are well described by the Boltzmann equation $[1,2,3,4]$, but the solution of the Boltzmann equation is numerically expensive. Macroscopic transport equations for rarefied gas flows promise numerically cheaper simulations of rarefied flows.

Macroscopic equations can be derived from the Boltzmann equation by several methods. The classical Chapman-Enskog method yields the Burnett and superBurnett equations [3, 2, 4], which, however, are unstable $[5,6]$, and thus cannot be used in practical applications. Another classical approach is Grad's moment method which in particular yields the well-known 13 moment equations $[7,8]$. In the last decade several authors developed other models, including the augmented Burnett equations [9], the regularized Burnett equations [10], the hyperbolic Burnett equations [11], consistently ordered extended thermodynamics [12], and the regularized 13 moment equations (R13 equations) [13, 14]. The R13 equations can be derived by a careful consideration of the Knud- sen order of magnitude of all terms appearing in the equations $[15,16]$, they are of super-Burnett order and avoid the stability problems associated with the Burnett-type equations. The same ordering method shows that Grad's 13 moment equations are the proper equations of second order for Maxwell molecules [15], while one obtains a variant of the Grad equations for other molecule types [16, 4].

The different methods to derive the equations and the merits and problems of the various sets of equations are discussed in the cited references, see Ref. [4] for a comprehensive review. All of the mentioned sets of equations go beyond the Navier-Stokes-Fourier (NSF) system, and include the NSF equations in the limit of small Knudsen numbers.

The solution of the NSF equations requires boundary conditions for velocities and temperature, while the higher order models require additional boundary conditions for gradients of velocity and temperature, or for stresses and heat flux. The derivation of meaningful boundary conditions is a major obstacle that needs to be overcome in order to render any of the higher order models into a useful engineering tool. Since the macroscopic equations follow

Copyright (c) 200x Inderscience Enterprises Ltd. 
from the Boltzmann equation, one will expect that the boundary conditions for the macroscopic equations can be derived from the boundary conditions for the Boltzmann equation, which are readily available [1].

In the present paper we discuss this problem for Grad's 13 moment equations applied to Couette flow. The solution of the equations requires jump and slip boundary conditions for temperature and velocity and additional boundary conditions for the normal stress. We shall discuss how these can be derived from the boundary conditions for the Boltzmann equation. Our main results and simulations were presented in Thatchers masters thesis [17].

Under some restrictive assumptions one can solve Couette flow with Grad's equations with boundary conditions for temperature and velocity only. Marques and Kremer presented an analytical solution of the Grad 13 equations with jump and slip, under the additional condition of constant pressure [18]. Their results show good agreement with DSMC simulations for Knudsen numbers up to $\mathrm{Kn}=0.25$. Liu and Rincon, using an iterative approximation method, found a similar solution numerically [19]; they were not interested in incorporating jump and slip. Similar methods are presented in Refs. [20] and [21]. Solutions of the Burnett equations with ad-hoc boundary conditions for normal stresses were presented in [23]. The results differed considerably from exact solutions of the Boltzmann equation as the Knudsen number became larger, which indicates problems with the boundary conditions. An expansion of the Grad equations in Couette geometry yields the second order bulk equations that require only jump and slip boundary conditions [17, 22, 4].

The remainder of the paper is organized as follows. Section 2 introduces the 13 moment equations. The equations are reduced for the geometry of Couette flow. For comparison, the Navier-Stokes-Fourier (NSF) equations and the second order bulk equations are presented as well. Boundary conditions for the moment equations are discussed in Section 3. We present a standard derivation of the velocity slip and temperature jump boundary conditions, and use the same method to find an additional boundary condition for normal stresses. Numerical results are presented and discussed in Section 4, where we compare results to DSMC (Direct Simulation Monte Carlo) computations. The paper ends with our conclusions.

\section{Grad's 13 moment equations for Couette flow}

\subsection{Grad 13 equations}

We consider monatomic ideal gases described on the micro level by the phase density $f\left(x_{i}, t, c_{i}\right)$ which gives the number density of particles in the phase space element $d \mathbf{x} d \mathbf{c}$. Here, $x_{i}, t$ denote space and time variables, respectively, and $c_{i}$ is the velocity of a particle of mass $m$. The phase density is governed by the Boltzmann equation [1, 2, 3, 4],

$$
\frac{\partial f}{\partial t}+c_{k} \frac{\partial f}{\partial x_{k}}=\mathcal{S}(f)
$$

where the collision term $\mathcal{S}(f)$ accounts for the change of the phase density due to collisions among particles.

Once the phase density is known, one may calculate its moments, for instance the mass density $\rho$, the momentum density $\rho v_{i}$ and the density of internal energy $\rho \varepsilon$, given by

$$
\begin{aligned}
\rho & =m \int f d \mathbf{c}, \\
\rho v_{i} & =m \int c_{i} f d \mathbf{c}, \\
\rho \varepsilon & =\frac{3}{2} \rho \theta=\frac{3}{2} p=\frac{m}{2} \int C^{2} f d \mathbf{c} .
\end{aligned}
$$

Here, $\theta=\frac{k}{m} T$ is the temperature in energy units ( $k$ is Boltzmann's constant, $m$ is particle mass, $T$ is thermodynamic temperature) and $v_{i}$ is the macroscopic velocity of the gas. The pressure obeys the ideal gas law, $p=\rho \theta$ and $C_{i}=c_{i}-v_{i}$ is the peculiar velocity.

Grad's moment method [7] is based on the assumption that the state of the gas is satisfactorily described by a set $u_{A}=m \int \psi_{A} f d \mathbf{c}$ of moments of the phase density. Moment equations result from multiplying the Boltzmann equation by $\psi_{A}$ and integration over c. If the $\psi_{A}$ depend only on the microscopic velocity $c_{i}$ the moment equations assume balance type form,

$$
\frac{\partial u_{A}}{\partial t}+\frac{\partial F_{A k}}{\partial x_{k}}=P_{A}
$$

with

$$
F_{A k}=m \int \psi_{A} c_{k} f d \mathbf{c} \quad, \quad P_{A}=m \int \psi_{A} \mathcal{S}(f) d \mathbf{c} .
$$

The variables in Grad's 13 moment equations follow either from $\psi_{A}=\left\{1, c_{i}, \frac{1}{2} c^{2}, c_{\langle i} c_{j\rangle}, \frac{1}{2} c^{2} c_{i}\right\}$, or the equivalent choice $\bar{\psi}_{A}=\left\{1, C_{i}, \frac{1}{2} C^{2}, C_{\langle i} C_{j\rangle}, \frac{1}{2} C^{2} C_{i}\right\}$ [4]. Thus, the relevant variables in Grad's 13 moment theory are the hydrodynamic variables $\rho, v_{i}$, and $\varepsilon$ and the deviatoric pressure tensor $\sigma_{i j}$ (with $\sigma_{i j}=\sigma_{j i}$ and $\sigma_{k k}=0$ ), called stress from now on, and heat flux $q_{i}$, given by

$$
\sigma_{i j}=m \int C_{\langle i} C_{j\rangle} f d \mathbf{c} \quad, \quad q_{i}=\frac{m}{2} \int C^{2} C_{i} f d \mathbf{c} .
$$

Indices in angular brackets indicate the symmetric and trace-free part of a tensor [4].

The equations for the moments follow by multiplication of the Boltzmann equation with $\psi_{A}$ or $\bar{\psi}_{A}$ and subsequent integration over velocity space. The resulting equations contain the fluxes $F_{A k}$ and the collision integrals $P_{A}$ which are not a priori related to the moments. For the closure of the equations, Grad constructed a phase density by means of an expansion around local equilibrium, where the phase density is a Maxwellian $f_{M}$, as $[7,8]$

$$
f_{13}=f_{M}\left(1+\frac{\sigma_{j k}}{2 \rho \theta^{2}} C_{j} C_{k}-\frac{q_{k}}{\rho \theta^{2}} C_{k}\left(1-\frac{1}{5} \frac{C^{2}}{\theta}\right)\right) \text {. }
$$

with the Maxwellian

$$
f_{M}=\frac{\rho}{m} \sqrt{\frac{1}{2 \pi \theta}}^{3} e^{-\frac{C^{2}}{2 \theta}} .
$$


The resulting moment equations are the conservation laws for mass, momentum and energy,

$$
\begin{aligned}
\frac{D \rho}{D t}+\rho \frac{\partial v_{k}}{\partial x_{k}} & =0, \\
\rho \frac{D v_{i}}{D t}+\theta \frac{\partial \rho}{\partial x_{i}}+\rho \frac{\partial \theta}{\partial x_{i}}+\frac{\partial \sigma_{i k}}{\partial x_{k}} & =\rho G_{i} \\
\frac{3}{2} \rho \frac{D \theta}{D t}+\rho \theta \frac{\partial v_{k}}{\partial x_{k}}+\frac{\partial q_{k}}{\partial x_{k}}+\sigma_{k l} \frac{\partial v_{k}}{\partial x_{l}} & =0,
\end{aligned}
$$

plus the moment equations for stress (with the production terms for Maxwell molecules [4])

$$
\frac{D \sigma_{i j}}{D t}+\frac{4}{5} \frac{\partial q_{\langle i}}{\partial x_{j\rangle}}+2 \sigma_{k\langle i} \frac{\partial v_{j\rangle}}{\partial x_{k}}+\sigma_{i j} \frac{\partial v_{k}}{\partial x_{k}}+2 \rho \theta \frac{\partial v_{\langle i}}{\partial x_{j\rangle}}=-\frac{p}{\mu} \sigma_{i j},
$$

$$
\begin{aligned}
\frac{D q_{i}}{D t}+ & \frac{5}{2} \rho \theta \frac{\partial \theta}{\partial x_{i}}+\frac{5}{2} \sigma_{i k} \frac{\partial \theta}{\partial x_{k}}-\sigma_{i k} \theta \frac{\partial \ln \rho}{\partial x_{k}}-\frac{\sigma_{i k}}{\rho} \frac{\partial \sigma_{k l}}{\partial x_{l}}+\theta \frac{\partial \sigma_{i k}}{\partial x_{k}} \\
& +\frac{7}{5} q_{i} \frac{\partial v_{k}}{\partial x_{k}}+\frac{7}{5} q_{k} \frac{\partial v_{i}}{\partial x_{k}}+\frac{2}{5} q_{k} \frac{\partial v_{k}}{\partial x_{i}}=-\frac{2}{3} \frac{p}{\mu} q_{i}
\end{aligned}
$$

Here, $D / D t=\partial / \partial t+v_{k} \partial / \partial x_{k}$ is the material time derivative and $\mu=\mu_{0}\left(\theta / \theta_{0}\right)$ denotes the viscosity of Maxwell molecules; $\mu_{0}$ is a viscosity at reference temperature $\theta_{0}$. Note that we have written the equations for the moments of $\bar{\psi}_{A}$, in which the equations are not of the form (3).

By means of a Chapman-Enskog expansion to first order in the Knudsen number $\mathrm{Kn}=\frac{\mu \sqrt{\theta}}{p L}$ one can recover the laws of Navier-Stokes and Fourier [4],

$$
\sigma_{i j}=-2 \mu \frac{\partial v_{\langle i}}{\partial x_{j\rangle}} \quad, \quad q_{i}=-\frac{15}{4} \mu \frac{\partial \theta}{\partial x_{i}} .
$$

\subsection{Couette geometry and conservation laws}

Plane Couette flow is a simple steady state flow where rarefaction effects such as velocity slip, temperature jumps, and Knudsen boundary layers can be studied. Two infinite parallel plates at constant distance $L$ move with the constant velocities $v_{W}^{0}, v_{W}^{L}$ relative to each other in their respective planes, and are kept at constant temperatures $\theta_{W}^{0}, \theta_{W}^{L}$. The coordinate frame is chosen such that the planes move into the direction $x=x_{1}$, and $y=x_{2}$ is the direction perpendicular to the plates. We chose a frame where $v_{W}^{0}=0$ and assume equal wall temperatures, $\theta_{W}^{0}=\theta_{W}^{L}=\theta_{W}$.

Due to the symmetry of the problem, all variables will depend only on the coordinate $y$. Since the walls are impermeable, the velocity of the gas must point into the $x$ direction, that is

$$
v_{i}=\{v(y), 0,0\}_{i} \text { and thus } \frac{\partial v_{k}}{\partial x_{k}}=0, \frac{D}{D t}=0 .
$$

Furthermore, since the set-up is independent of the third space coordinate, $z=x_{3}$, neither stress nor heat flux should be associated with that direction, so that $\sigma_{13}=$

$$
\begin{aligned}
\sigma_{23}=q_{3} & =0, \text { and } \\
\sigma_{i j} & =\left[\begin{array}{ccc}
\sigma_{11}(y) & \sigma_{12}(y) & 0 \\
\sigma_{12}(y) & \sigma_{22}(y) & 0 \\
0 & 0 & -\sigma_{11}(y)-\sigma_{22}(y)
\end{array}\right]_{i j} \\
q_{i} & =\left\{q_{1}(y), q_{2}(y), 0\right\}_{i}
\end{aligned}
$$

The macroscopic description is based on the conservation laws (7) which here reduce to

$$
\begin{aligned}
0 & =0, \frac{d \sigma_{12}}{d y}=0, \frac{d \rho \theta}{d y}+\frac{d \sigma_{22}}{d y}=0, \\
\frac{d \sigma_{32}}{d y} & =0, \frac{d q_{2}}{d y}=-\sigma_{12} \frac{d v}{d y} .
\end{aligned}
$$

The first and fourth equation are trivial, and two of the remaining three can be integrated so that the conservation laws assume the form

$\sigma_{12}=$ const.,$\rho \theta+\sigma_{22}=P_{0}=$ const.,$q_{2}+\sigma_{12} v=$ const.

Three boundary conditions are required to determine the three constants of integration in (12).

The further evaluation depends on the laws considered for $\sigma_{i j}$ and $q_{i}$.

\subsection{Navier-Stokes-Fourier}

In Couette geometry, the NSF equations (10) reduce to

$$
\sigma_{12}=-\mu \frac{d v}{d y}, \quad q_{2}=-\frac{15}{4} \mu \frac{d \theta}{d y},
$$

all other components of stress $\sigma_{i j}$ and heat flux $q_{i}$ vanish. From (12) follows that the pressure is constant, $\rho \theta=P_{0}$, and the two other conservation laws assume the form

$$
-\mu \frac{d v}{d y}=\sigma_{12} \quad, \quad \mu \frac{d}{d y}\left(\mu \frac{d \theta}{d y}\right)=-\frac{4 \sigma_{12}^{2}}{15} ;
$$

recall that by Eq. (12) $\sigma_{12}$ is constant. The solution of these equations requires jump and slip boundary conditions for temperature and velocity. Moreover, an additional condition is required to determine the pressure $P_{0}$; this constant follows from the constant mass (per unit area) between the walls as, see $(12)_{2}$,

$$
M=\int_{0}^{L} \rho d y=P_{0} \int_{0}^{L} \frac{d y}{\theta} .
$$

\subsection{Grad 13 equations for Couette flow}

The Grad 13 equations in Couette geometry read

$$
\begin{aligned}
P_{0} \frac{d v}{d y}+\frac{2}{5} \frac{d q_{1}}{d y} & =-\frac{\rho \theta}{\mu} \sigma_{12}, \\
-\frac{6}{5} \sigma_{12} \frac{d v}{d y} & =-\frac{\rho \theta}{\mu} \sigma_{22}, \\
\frac{7}{5} q_{2} \frac{d v}{d y}+\frac{7}{2} \sigma_{12} \frac{d \theta}{d y} & =-\frac{2}{3} \frac{\rho \theta}{\mu} q_{1}, \\
\frac{2}{5} q_{1} \frac{d v}{d y}+\left(\frac{5}{2} P_{0}+\sigma_{22}\right) \frac{d \theta}{d y}+\theta \frac{d \sigma_{22}}{d y} & =-\frac{2}{3} \frac{\rho \theta}{\mu} q_{2} .
\end{aligned}
$$


Moreover, one finds $\sigma_{11}=-\frac{4}{3} \sigma_{22}$.

The determinant of the matrix of coefficients in (16) is

$$
\operatorname{det}\left[\begin{array}{cccc}
P_{0} & 0 & 0 & \frac{2}{5} \\
-\frac{6}{5} \sigma_{12} & 0 & 0 & 0 \\
\frac{7}{5} q_{2} & \frac{7}{2} \sigma_{12} & 0 & 0 \\
\frac{2}{5} q_{1} & \frac{5}{2} P_{0}+\sigma_{22} & \theta & 0
\end{array}\right]=\frac{42}{25} \sigma_{12}^{2} \theta .
$$

This is non-zero, as long as $\sigma_{12} \neq 0$. Therefore the differential equations are linearly independent, and their solution requires seven boundary conditions: four for (16) plus three for the conservation laws (12). We shall consider jump and slip boundary conditions for temperature and velocity, and additional boundary conditions for normal stress $\sigma_{22}$. The constant $P_{0}$ follows again from the constant mass between the walls as, see $(12)_{2}$,

$$
M=\int_{0}^{L} \rho d y=\int_{0}^{L} \frac{P_{0}-\sigma_{22}}{\theta} d y .
$$

Grad, in Ref. [8], states that the linearized 13 moment equations need as many boundary conditions as the NSF equations. His argument is based on the characteristic speeds of the linearized equations, which have 6 non-zero roots and a triple zero root. Only non-zero characteristics require boundary conditions, and Grad states that these are: $(1,2)$ slip at both walls, $(3,4)$ temperature jump at both walls, (5) average mass between the walls, (6) zero normal velocity (this was imposed in the steady state equations above already).

Indeed, linearizing the Grad 13 equations for steadystate Couette flow (16) over an equilibrium reference state given by $\rho_{0}, \theta_{0}$, and $v_{i \mid 0}=\sigma_{i j \mid 0}=q_{i \mid 0}=0$ yields

$$
\sigma_{12}=-\mu_{0} \frac{d v}{d y}, \quad \sigma_{22}=0, \quad q_{1}=0, \quad q_{2}=-\frac{15}{4} \mu_{0} \frac{d \theta}{d y} .
$$

These are just the (linearized) NSF equations for the Couette flow problem. This shows that also in steady state the linearized Grad equations require as many boundary conditions as NSF.

In this paper, however, we consider the non-linear 13 moment equations and these require two additional boundary conditions. While we did not compute the characteristic speeds of the non-linear time-dependent system, we infer that the non-linear equations have 8 non-zero characteristic speeds, and only one zero root. In linearization, two of the non-zero roots degenerate to zero roots.

\subsection{Bulk equations}

When the Grad 13 moment equations for Couette flow are considered only within second order terms in the Knudsen number, they can be reduced to the so-called bulk equations $[17,22,4]$,

$$
\begin{aligned}
\sigma_{12} & =-\mu \frac{\partial v}{\partial y}, \sigma_{22}=-\frac{6}{5} \frac{\sigma_{12} \sigma_{12}}{P_{0}} \\
q_{1} & =\frac{7}{2} \frac{\sigma_{12} q_{2}}{P_{0}}, \quad q_{2}=-\frac{15}{4} \mu \frac{d \theta}{d y} .
\end{aligned}
$$

These are the essentially NSF equations with two additional equations for normal stress $\sigma_{22}$ and parallel heat flux $q_{1}$. The solution of the bulk equations requires jump and slip boundary conditions for temperature and velocity, but no additional boundary conditions for normal stress. Indeed, one can solve the NSF system first (with second order jump and slip conditions), and then use the result to compute the rarefaction quantities $\sigma_{22}$ and $q_{1}$. Note in particular that the heat flux $q_{1}$ is not driven by a temperature gradient.

The bulk equations can also be found from the Burnett equations under omission of some higher order terms, see the appendix of Ref. [22] for details. The heat flux parallel to the wall is reported in Ref. [18] with a different coefficient, as $\frac{39}{5} \frac{\sigma_{12} q_{2}}{P_{0}}$, probably due to an oversight in the derivation.

\section{Jump and slip boundary conditions}

\subsection{Basic considerations}

The simplest model for the boundary conditions of the Boltzmann equation is due to Maxwell. While more refined models are available, Maxwell's boundary conditions are widely used, and are well suited for our study. According to Maxwell, the boundary condition for the phase density reads

$\bar{f}= \begin{cases}\chi f_{W}+(1-\chi) f_{N}\left(-C_{k}^{W} n_{k}\right) & , \quad C_{k}^{W} n_{k} \geq 0 \\ f_{N}\left(C_{k}^{W} n_{k}\right) & , \quad C_{k}^{W} n_{k} \leq 0\end{cases}$

Here, $\chi$ is the accommodation coefficient, $n_{k}$ is the wall normal pointing into the gas, and

$$
f_{W}=\frac{\rho_{W}}{m}{\sqrt{\frac{1}{2 \pi \theta_{W}}}}^{3} \exp \left[-\frac{C_{W}^{2}}{2 \theta_{W}}\right]
$$

is the wall Maxwellian; $f_{N}$ denotes the phase density of the incoming particles $\left(C_{k}^{W} n_{k} \leq 0\right)$ and $f_{N}\left(-C_{k}^{W} n_{k}\right)$ denotes the distribution of specularly reflected particles. The boundary condition state that the fraction $\chi$ of the particles that hit the wall thermalize, and leave the wall in a Maxwellian distribution (described by temperature and velocity of the wall), while the remaining fraction is specularly reflected.

We use Fig. 1 to study the boundary conditions on the level of moments. For this we integrate the moment equation for a moment $u_{A}=\int \psi_{A} f d \mathbf{c}$ in divergence form (3) over the volume $\Delta V=\Delta A \Delta x$, to obtain

$$
\frac{d}{d t} \int_{\Delta V} u_{A} d V+\oint_{\partial \Delta V} F_{A k} \bar{n}_{k} d A=\int_{\Delta V} P_{A} d V,
$$

where $\partial \Delta V$ denotes the surface of $\Delta V$, and $\bar{n}_{k}$ its outer normal. For the following, we assume that the surface element $\Delta A$ is so small that moments and fluxes do not vary on $\Delta A$. The volume integrals vanish in the limit 


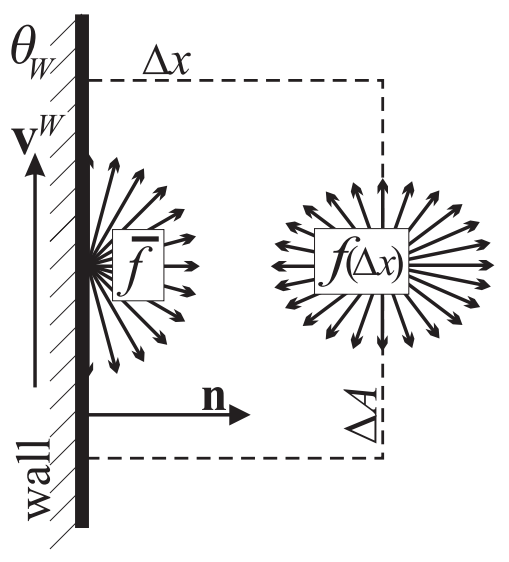

Figure 1: The phase density in front of a wall at temperature $\theta_{W}$ with velocity $v_{W} . f(\Delta x)$ denotes the phase density in distance $\Delta x$ from the wall.

$\Delta x \rightarrow 0$ which thus gives

$$
\left(\bar{F}_{A k}-F_{A k}^{N}\right) n_{k} \Delta A=0 .
$$

The fluxes are defined in the rest frame of the wall, as

$$
\bar{F}_{A k}=\int \psi_{A} C_{k}^{W} \bar{f} d \mathbf{c}, F_{A k}^{N}=\lim _{\Delta x \rightarrow 0} \int \psi_{A} C_{k}^{W} f(\Delta x) d \mathbf{c} .
$$

Any accurate solution of the Boltzmann equation with the Maxwell boundary condition will fulfill condition (23) for all moments, since by definition $\lim _{\Delta x \rightarrow 0} f(\Delta x)=f_{N}$ (for $\left.C_{k}^{W} n_{k} \leq 0\right)$.

The collisions between particles and wall change the phase density, so that the distributions of incoming and outgoing particles will be very different. Most importantly, the phase density directly at the wall, $\bar{f}$, will be discontinuous in velocity space unless thermal equilibrium prevails. Collisions will relax this discontinuity within a layer of few mean free paths away from the wall, the so-called Knudsen layer.

Grad's distribution (5) is not well suited to describe the discontinuous distribution (21) at the wall, and one will expect some difficulties for the Grad equations to describe the gas close to the wall, and in the Knudsen layer. Nevertheless, we shall assume $f_{N}=f_{13}$.

The particle velocity $c_{i}$, the particle velocity relative to the wall $C_{i}^{W}$, and the peculiar velocity $C_{i}$ are related by

$C_{i}^{W}=c_{i}-v_{i}^{W}=C_{i}+V_{i}, C_{i}^{W} n_{i}=c_{i} n_{i}-v_{i}^{W} n_{i}=C_{i} n_{i}$.

$V_{i}=v_{i}-v_{i}^{W}$ denotes the slip velocity, which is parallel to the wall, $V_{k} n_{k}=0$.

The computations for this chapter require half-space integrals of Maxwellian distributions and of Grad's 13 moment distribution which can be found in [24, 4]. Gas particles do not accumulate at the walls, and the corresponding condition for conservation of mass follows from (23) for $\psi_{A}=1$, as

$$
\rho_{W}=\frac{p+\frac{1}{2} \sigma_{i k} n_{i} n_{k}}{\sqrt{\theta_{W} \theta}} .
$$

To find boundary conditions we consider Eq. (23) for the following choices for $\psi$ :

(a) momentum parallel to the wall, measured in the rest frame of the wall,

$$
\psi_{c_{i}}^{\|}=C_{i}^{W}-n_{i} C_{r}^{W} n_{r}=C_{i}-n_{i} C_{r} n_{r}+V_{i},
$$

(b) energy

$$
\psi_{\frac{1}{2} c^{2}}=\frac{m}{2} C_{W}^{2}=\frac{m}{2}\left(C^{2}+2 V_{i} C_{i}+V^{2}\right),
$$

(c) momentum perpendicular to the wall, measured in the rest frame of the wall

$$
\psi_{c_{i}}^{\perp}=C_{r}^{W} n_{r}=C_{r} n_{r}
$$

The resulting jump and slip conditions are

$$
\begin{aligned}
V_{i}= & \frac{-\frac{2-\chi}{\chi} \sqrt{\frac{\pi}{2}} \sqrt{\theta}\left(\sigma_{i k} n_{k}-n_{i} \sigma_{j k} n_{j} n_{k}\right)}{\rho \theta+\frac{1}{2} \sigma_{i k} n_{i} n_{k}} \\
-\frac{\frac{1}{5}\left(q_{i}-n_{i} n_{j} q_{j}\right)}{\rho \theta+\frac{1}{2} \sigma_{i k} n_{i} n_{k}} & (26) \\
\theta-\theta_{W}= & \frac{V^{2}}{4}-\frac{\frac{2-\chi}{\chi} \frac{1}{2} \sqrt{\frac{\pi}{2}} \sqrt{\theta} q_{k} n_{k}+\frac{1}{4} \theta \sigma_{i k} n_{i} n_{k}}{\rho \theta+\frac{1}{2} \sigma_{i k} n_{i} n_{k}}, \\
\sigma_{i k} n_{i} n_{k}= & \frac{-\frac{2-\chi}{\chi} \frac{2}{5} \sqrt{\frac{2}{\pi}} q_{k} n_{k}+\left(\sqrt{\theta_{W}}-\sqrt{\theta}\right) \rho \theta}{\sqrt{\theta}-\frac{1}{2} \sqrt{\theta_{W}}} .
\end{aligned}
$$

Note that the tangential stress $\left(\sigma_{i k} n_{k}-n_{i} \sigma_{k r} n_{k} n_{r}\right)$ and the normal heat flux $q_{k} n_{k}$ vanish for $\chi \rightarrow 0$ - there are no shear stresses at an elastically reflecting wall, and it is adiabatic. The equations $(26)_{1,2}$ can be found in Ref. [8], Eqs. $(29.27,29.29)$ with $a_{M}=\alpha_{E}$, where, however, the term $\frac{1}{4} V^{2}$ is missing. This term describes a significant contribution to the temperature jump that needs to be considered in a second order theory $[22,4]$.

\subsection{Knudsen layer correction}

As stated above, it cannot be expected that the Grad distribution gives an accurate description of the gas close to the wall, i.e. in the Knudsen layer. Accordingly, the calculations of jump and slip conditions will not be exact. The simplest way to account for the additional contributions is to introduce correction factors $\alpha_{\omega}, \beta_{\omega}, \gamma_{\omega}(\omega=1,2)$ into the boundary conditions, so that

$$
\begin{aligned}
& V_{i}= \frac{-\alpha_{1} \frac{2-\chi}{\chi} \sqrt{\frac{\pi}{2}} \sqrt{\theta}\left(\sigma_{i k} n_{k}-n_{i} \sigma_{j k} n_{j} n_{k}\right)}{\rho \theta+\frac{1}{2} \sigma_{i k} n_{i} n_{k}} \\
&\left.-\frac{\alpha_{2} \frac{1}{5}\left(q_{i}-n_{i} n_{j} q_{j}\right)}{\rho \theta+\frac{1}{2} \sigma_{i k} n_{i} n_{k}}, \quad 27\right) \\
& \theta-\theta_{W}=\frac{V^{2}}{4}-\frac{\beta_{1} \frac{2-\chi}{\chi} \frac{1}{2} \sqrt{\frac{\pi}{2}} \sqrt{\theta} q_{k} n_{k}+\beta_{2} \frac{1}{4} \theta \sigma_{i k} n_{i} n_{k}}{\rho \theta+\frac{1}{2} \sigma_{i k} n_{i} n_{k}}, \\
& \sigma_{i k} n_{i} n_{k}=\frac{-\gamma_{1} \frac{2-\chi}{\chi} \frac{2}{5} \sqrt{\frac{2}{\pi}} q_{k} n_{k}+\gamma_{2} p\left(\sqrt{\theta_{W}}-\sqrt{\theta}\right)}{\sqrt{\theta}-\frac{1}{2} \sqrt{\theta_{W}}} .
\end{aligned}
$$


The derivation of the previous section, which ignores Knudsen layer corrections, gives $\alpha_{1}=\alpha_{2}=\beta_{1}=\beta_{2}=1$. Many authors have computed these coefficients for the NSF equations, mostly from numerical solutions of the Boltzmann equation, or of related kinetic models, see, e.g., [25]. Sharipov and Seleznev give a detailed overview of the literature [26, 27]. Typical values for the coefficients, computed with diffusive boundary conditions, i.e. $\chi=1$, are $[27,25]$

$$
\alpha_{1}=1.114 \quad, \quad \alpha_{2}=1.34533 \quad, \quad \beta_{1}=1.127 \text {. }
$$

Values for $\beta_{2}$ are not available, and we shall use $\beta_{2}=1$. Considering the relative simplicity of the above argument, it is remarkably successful, since it describes the effects leading to slip and temperature jump quite well.

\subsection{Maxwell-Smoluchowski boundary conditions}

In order to obtain jump and slip conditions for the NavierStokes-Fourier theory, stress and heat flux must be replaced by the NSF laws (10). To simplify notation, the derivative in normal direction is introduced as $\frac{\partial}{\partial n}=n_{k} \frac{\partial}{\partial x_{k}}$, and the tangential derivative as $\frac{\partial}{\partial \tau_{i}}=\frac{\partial}{\partial x_{i}}-n_{i} \frac{\partial}{\partial n}$. Moreover, the velocity is split into its tangential and normal parts, $v_{i}=v_{i}^{\tau}+n_{i} v_{n}$. Note that $v_{n}=0$ directly at the wall, which implies $\frac{\partial v_{n}}{\partial \tau_{k}}=0$, but not $\frac{\partial v_{n}}{\partial n}=0$.

Since the NSF equations are of first order in the Knudsen number, normally only the leading order terms of (27) are considered,

$$
V_{i}=\frac{2-\chi}{\chi} \alpha_{1} \sqrt{\frac{\pi}{2}} \frac{\mu}{\rho \sqrt{\theta}} \frac{\partial v_{i}^{\tau}}{\partial n}+\frac{\alpha_{2}}{2 \operatorname{Pr}} \frac{\mu}{\rho \theta} \frac{\partial \theta}{\partial \tau_{i}},
$$

$\theta-\theta_{W}=\frac{2-\chi}{\chi} \frac{5 \beta_{1}}{4 \operatorname{Pr}} \sqrt{\frac{\pi}{2}} \frac{\mu}{\rho \sqrt{\theta}} \frac{\partial \theta}{\partial n}+\beta_{2} \frac{1}{6} \frac{\mu}{\rho}\left(2 \frac{\partial v_{n}}{\partial n}-\frac{\partial v_{r}^{\tau}}{\partial \tau_{r}}\right)$

These are the well-known Maxwell-Smoluchowski jump and slip boundary conditions for the NSF equations. A slip velocity occurs due to a velocity gradient normal to the wall (classical slip), or due to a temperature gradient along the wall (thermal creep, thermal transpiration [28]). Recently it was suggest to include higher order contributions into the jump and slip boundary conditions for NSF [29].

A temperature jump results from a temperature gradient perpendicular to the wall, or due to velocity gradients. The velocity effects are usually ignored in the literature, probably because they are very small, and cannot be controlled in experiments.

\subsection{NSF solution (as reference)}

We solve the NSF equations for Couette flow, where the boundary conditions (28) reduce to

$$
\begin{aligned}
v-v_{W}^{\alpha} & =\frac{2-\chi}{\chi} \alpha_{1} \sqrt{\frac{\pi}{2}} \frac{\mu \sqrt{\theta}}{P_{0}} \frac{d v}{d y} n^{\alpha}, \\
\theta-\theta_{W} & =\frac{2-\chi}{\chi} \frac{15}{8} \beta_{1} \sqrt{\frac{\pi}{2}} \frac{\mu \sqrt{\theta}}{P_{0}} \frac{d \theta}{d y} n^{\alpha},
\end{aligned}
$$

with $\alpha=0, L$ and $n^{0}=-n^{L}=1$. Recall that the viscosity is a function of temperature alone, $\mu=\mu(\theta)$.

We consider a somewhat linearized version of the equations by assuming only small temperature variations around $\theta_{W}$. Then the viscosity can be assumed to be constant, $\mu=\mu\left(\theta_{W}\right)$, and the equations read

$$
\frac{d v}{d y}=a=\text { const. } \quad, \quad \frac{d^{2} \theta}{d y^{2}}=-\frac{4}{15} a^{2},
$$

with the linearized boundary conditions

$$
\begin{aligned}
v-v_{W}^{\alpha} & =\frac{2-\chi}{\chi} \alpha_{1} \sqrt{\frac{\pi}{2}} \operatorname{Kn} a L n^{\alpha}, \\
\theta-\theta_{W} & =\frac{2-\chi}{\chi} \frac{15}{8} \beta_{1} \sqrt{\frac{\pi}{2}} \operatorname{Kn} L \frac{d \theta}{d y} n^{\alpha},
\end{aligned}
$$

where $\mathrm{Kn}=\mu\left(\theta_{W}\right) \sqrt{\theta_{W}} /\left(P_{0} L\right)$ is the Knudsen number.

The equations (30) with the boundary conditions (31) are easy to integrate, and the result can be written as

$$
\begin{aligned}
v & =\frac{v_{w}^{L}}{2}+a\left(\frac{y}{L}-\frac{1}{2}\right) \quad, a=\frac{\left(v_{w}^{L}-v_{w}^{0}\right)}{1+\frac{2-\chi}{\chi} \sqrt{2 \pi} \alpha_{1} \mathrm{Kn}}, \\
\theta & =\theta_{W}+\frac{2-\chi}{\chi} \frac{\beta_{1}}{4} \sqrt{\frac{\pi}{2}} \mathrm{Kn} a^{2}+\frac{\operatorname{Pr}}{5} a^{2} \frac{y}{L}\left(1-\frac{y}{L}\right) \\
\sigma_{12} & =-\mathrm{Kn} \frac{P_{0} a}{\sqrt{\theta_{W}}}, q_{2}=\mathrm{Kn} \frac{P_{0} a^{2}}{\sqrt{\theta_{W}}}\left(\frac{y}{L}-\frac{1}{2}\right)
\end{aligned}
$$

The solutions are symmetric with respect to $y=\frac{L}{2}$, velocity $v$ and heat flux $q_{2}$ are straight lines, while the temperature $\theta$ is a parabola, and, of course, $\sigma_{12}$ is a constant. The temperature rises due to heating by friction, and is largest at $y=\frac{L}{2}$.

It is instructive to study temperature jump and slip in this case. Due to the symmetry, it suffices to consider both at $y=0$, where

$$
\begin{aligned}
v^{0}-v_{W}^{0} & =\frac{2-\chi}{\chi} \sqrt{\frac{\pi}{2}} \alpha_{1} \operatorname{Kn} a \\
\theta^{0}-\theta_{W}^{0} & =\frac{9}{16} \frac{2-\chi}{\chi} \sqrt{\frac{\pi}{2}} \beta_{1} \mathrm{Kn} a^{2} .
\end{aligned}
$$

Obviously, the Knudsen number strongly affects jump and slip. In the limit $\mathrm{Kn} \rightarrow 0$, jump and slip vanish, $v^{0}=v_{W}^{0}$, $\theta^{0}=\theta_{W}^{0}$. As the Knudsen number grows, the slip grows and approaches $\frac{v_{w}^{L}-v_{w}^{0}}{2}$ as $\mathrm{Kn} \rightarrow \infty$. In the latter case, the velocity is constant throughout the domain, $a=0$, which reflects that particles do not interact anymore. Thus, there is no frictional heating $\left(q_{2}=0\right)$, and the temperature is constant at $\theta_{W}$.

At small Knudsen numbers, jump and slip can be ignored, but they play a considerable role as the Knudsen number grows. Note also their dependence on the shear rate $a$.

The bulk equations (20) can be used to compute the second order (in $\mathrm{Kn}$ ) contributions to $\sigma_{22}$ and $q_{1}$ under the same simplifying assumptions as

$$
\sigma_{22}=-\frac{6}{5} \mathrm{Kn}^{2} \frac{P_{0} a^{2}}{\theta_{W}}, q_{1}=-\frac{7}{2} \mathrm{Kn}^{2} \frac{P_{0} a^{3}}{\theta_{W}}\left(\frac{y}{L}-\frac{1}{2}\right) \text {. }
$$


We emphasize that both quantities are zero for the NSF equations, their non-zero values describe rarefaction effects which become only visible when the Knudsen number is not small. Note also the dependence on the shear rate $a$ which indicates that $\sigma_{22}$ and $q_{1}$ might assume measurable values for large shear rates, even at smaller Knudsen numbers.

\section{5 $\sigma_{n n}$ boundary condition}

From the Chapman-Enskog expansion and the bulk solution it is known that for Couette flow $q_{2}$ and $\sigma_{12}$ are of first order in the Knudsen number and $q_{1}$ and $\sigma_{22}$ are of second order. This implies, as stated before, that for Couette flow with the NSF equations one finds $\sigma_{22}=0$. The boundary condition for $\sigma_{22}$ should be in accordance with this, that is the boundary condition $(27)_{3}$ should be of second order. This requirement can be used to determine one of the correction parameters $\gamma_{1}, \gamma_{2}$.

We proceed by expanding the boundary conditions in the Knudsen number. For this, the order of magnitude of the moments is made explicit by writing $\left\{\varepsilon \sigma_{12}, \varepsilon q_{2}, \varepsilon^{2} \sigma_{22}, \varepsilon^{2} q_{1}\right\}$ instead of $\left\{\sigma_{12}, q_{2}, \sigma_{22}, q_{1}\right\}$, where $\varepsilon$ is a scaling parameter that stands for the Knudsen number (the Knudsen number would appear in a dimensionless formulation, see [4]). Then, in Couette geometry, the boundary conditions (27) read

$$
\begin{aligned}
V & =\frac{-\frac{2-\chi}{\chi} \alpha_{1} \sqrt{\frac{\pi}{2} \theta} \varepsilon \sigma_{12} n_{2}-\frac{\alpha_{2}}{5} \varepsilon^{2} q_{1}}{p+\frac{1}{2} \varepsilon^{2} \sigma_{22}} \\
\theta-\theta_{W} & =\frac{V^{2}}{4}-\frac{\frac{2-\chi}{\chi} \frac{\beta_{1}}{2} \sqrt{\frac{\pi}{2} \theta} \varepsilon q_{2} n_{2}+\frac{\beta_{2}}{4} \theta \varepsilon^{2} \sigma_{22}}{p+\frac{1}{2} \varepsilon^{2} \sigma_{22}}, \quad \\
\varepsilon^{2} \sigma_{22} & =\frac{-\frac{2-\chi}{\chi} \frac{2 \gamma_{1}}{5} \sqrt{\frac{2}{\pi}} \varepsilon q_{2} n_{2}+\gamma_{2} p\left(\sqrt{\theta_{W}}-\sqrt{\theta}\right)}{\sqrt{\theta}-\frac{1}{2} \sqrt{\theta_{W}}} .
\end{aligned}
$$

These equations are now expanded to second order in $\varepsilon$; the jump and slip conditions $(35)_{1,2}$ reduce to

$$
\begin{aligned}
& V=-\varepsilon \frac{2-\chi}{\chi} \alpha_{1} \sqrt{\frac{\pi}{2}} \theta \frac{\sigma_{12} n_{2}}{p}-\varepsilon^{2} \alpha_{2} \frac{1}{5} \sqrt{\theta} \frac{q_{1}}{p \sqrt{\theta}}, \\
& \frac{\theta_{W}}{\theta}=1+\varepsilon \frac{2-\chi}{\chi} \frac{\beta_{1}}{2} \sqrt{\frac{\pi}{2}} \frac{q_{2} n_{2}}{p \sqrt{\theta}}+\varepsilon^{2} \frac{\beta_{2}}{4} \frac{\sigma_{22}}{p} \\
&-\varepsilon^{2}\left(\frac{2-\chi}{\chi}\right)^{2} \alpha_{1}^{2} \frac{\pi}{8}\left(\frac{\sigma_{12} n_{2}}{p}\right)^{2} .
\end{aligned}
$$

By expanding the square root of the second equation we find

$$
\begin{aligned}
& \sqrt{\frac{\theta_{W}}{\theta}}=1+\varepsilon \frac{2-\chi}{\chi} \frac{\beta_{1}}{4} \sqrt{\frac{\pi}{2}} \frac{q_{2} n_{2}}{p \sqrt{\theta}}+\varepsilon^{2} \frac{\beta_{2}}{8} \frac{\sigma_{22}}{p} \\
& -\varepsilon^{2}\left(\frac{2-\chi}{\chi}\right)^{2} \frac{\pi}{16}\left[\alpha_{1}^{2}\left(\frac{\sigma_{12} n_{2}}{p}\right)^{2}+\frac{\beta_{1}^{2}}{4}\left(\frac{q_{2} n_{2}}{p \sqrt{\theta}}\right)^{2}\right]
\end{aligned}
$$

which then is used in the expansion of the equation for $\sigma_{22}$ to obtain

$$
\begin{gathered}
\varepsilon^{2} \frac{\sigma_{22}}{p}=\varepsilon \frac{2-\chi}{\chi} \frac{4}{5} \sqrt{\frac{2}{\pi}}\left[\frac{5 \pi}{16} \gamma_{2} \beta_{1}-\gamma_{1}\right] \times \\
\frac{q_{2} n_{2}}{p \sqrt{\theta}}\left(1+\varepsilon \frac{2-\chi}{\chi} \frac{\beta_{1}}{4} \sqrt{\frac{\pi}{2}} \frac{q_{2} n_{2}}{p \sqrt{\theta}}\right) \\
+\varepsilon^{2} \gamma_{2}\left\{\frac{\beta_{2}}{4} \frac{\sigma_{22}}{p}\right\} \\
-\varepsilon^{2} \gamma_{2}\left\{\left(\frac{2-\chi}{\chi}\right)^{2} \frac{\pi}{8}\left[\alpha_{1}^{2}\left(\frac{\sigma_{12} n_{2}}{p}\right)^{2}+\frac{\beta_{1}^{2}}{4}\left(\frac{q_{2} n_{2}}{p \sqrt{\theta}}\right)^{2}\right]\right\}
\end{gathered}
$$

The first order contributions vanish when the square bracket in the first line is zero, that is for

$$
\gamma_{1}=\frac{5 \pi}{16} \gamma_{2} \beta_{1}
$$

With this choice, the leading (i.e. second order in $\mathrm{Kn}$ ) term of the boundary condition for $\sigma_{22}$ becomes

$$
\begin{aligned}
\frac{\sigma_{22}}{p}=-\left(\frac{2-\chi}{\chi}\right)^{2} \frac{\pi}{2} \frac{\gamma_{2} \alpha_{1}^{2}}{4-\gamma_{2} \beta_{2}} \times & \\
& {\left[\left(\frac{\sigma_{12} n_{2}}{p}\right)^{2}+\frac{\beta_{1}^{2}}{4 \alpha_{1}^{2}}\left(\frac{q_{2} n_{2}}{p \sqrt{\theta}}\right)^{2}\right] . }
\end{aligned}
$$

The complete boundary condition for $\sigma_{22}$ follows from Eq. $(35)_{3}$ together with the relation (36) as

$$
\sigma_{22}=\gamma \frac{-\frac{2-\chi}{\chi} \frac{1}{4} \sqrt{\frac{\pi}{2}} \beta_{1} q_{2} n_{2}+p\left(\sqrt{\theta_{W}}-\sqrt{\theta}\right)}{\sqrt{\theta}-\frac{1}{2} \sqrt{\theta_{W}}}
$$

where we replaced $\gamma_{2}=\gamma$.

\section{Computations}

\subsection{Numerical method}

Grad's 13 moment equations form a hyperbolic system of PDE's. In order to solve them with slip and jump conditions for velocity, temperature and normal stress we use a method provided by Liotta et al. [31]. These authors extended the Nessyahu-Tadmor scheme for hyperbolic conservation laws [32] to balance laws of relaxation type.

Steady state solutions were obtained by time stepping into equilibrium. The method requires the time dependent equations in balance law form (3), the appropriate form of the Grad equations for Couette flow is given in Appendix A. Note that, in order to have proper solutions, normal velocity $v_{2}$ (which is zero in steady state) and stress $\sigma_{11}$ (which is equal to $-\frac{4}{3} \sigma_{22}$ in steady state) must be included into the set of independent variables.

Moments that do not have explicit boundary conditions were assumed to follow the moment equations. For these, the only change that needed to be made was to ignore the method's minmod-reconstruction at the boundary and 
chose the finite difference immediately next to the boundary [17].

The numerical results presented below were obtained on a grid with 100 space steps. As recommended in [31] the time step was chosen (by trial and error) as large as possible, close to the stability limit. We used the explicit version of the scheme (UCS2), which requires small time steps. The computations require about 100 seconds on a 1.7 GHz Laptop.

Bulk equations and NSF equations were solved by means of a finite volume scheme [17].

\subsection{Non-linear transition layer vs. Knudsen layer}

The computational results depend on the factor $\gamma$ in Eq. (38). Figure 2 shows the results for the choice $\gamma=1.3$ with Knudsen number $\mathrm{Kn}=0.05$ and a velocity difference of $v_{W}^{L}-v_{W}^{0}=400 \frac{\mathrm{m}}{\mathrm{s}}$. The individual plots show results for the important variables according to the numerical solution, compared to DSMC solutions and a solution of the bulk equations (20) with second order jump and slip boundary conditions.

We observe an excellent agreement between all three curves for density, velocity, temperature, and the heat flux towards the wall, $q_{2}$. The differences in the data for $\sigma_{12}$, $\sigma_{22}, p$ are slightly bigger, but below $2.5 \%$. The DSMC data shows marked Knudsen layers, in particular in the results for the heat flux parallel to the wall, $q_{1}$, and the normal stress, $\sigma_{22}$. Bulk solution and numerical solution of Grad13 agree well with DSMC data outside the Knudsen boundary layers, i.e. in the bulk.

The numerical results for the Grad 13 equations show very narrow boundary layers as well. These transition layers are not Knudsen layers. Indeed, Knudsen layers usually are obtained as solutions of the linearized Boltzmann equation [1, 28, 4]. A typical Knudsen layer is of the form $A \exp \left[-b \frac{x}{\lambda}\right]$, where $A$ is an amplitude, $x$ is the distance from the wall, $\lambda$ is the mean free path, and $b$ is a number of order unity. Most macroscopic models give some linear Knudsen layers, but only the R13 equations give a complete set of Knudsen layers for velocity, temperature, stress and heat flux [4]. The Burnett models give linear Knudsen layers only for some quantities and also lead to nonphysical oscillatory solutions [30, 22, 4]. Grad's 13 moment equations, however, do not describe linear Knudsen layers. This can be seen by considering the linearized equations (19), which agree with the linearized NSF equations: both have no Knudsen layers. The rarefaction effects that are described by the Grad 13 equations are due to non-linear contributions.

Comparison between the Knudsen layers in the DSMC simulations and the transition layers in the Grad13 solution clearly shows that the Knudsen layers are markedly wider than the transition layers. We must consider the transition layers as artefacts of the Grad 13 equations (and their boundary conditions), not as effects that relate to the actual physics.

\subsection{Results for $\gamma=1.5$}

Since the boundary value for $\sigma_{22}$, Eq. (38), depends on the value of the coefficient $\gamma$, the size of the transition layers depends on $\gamma$. Different values of $\gamma$ produce different results. Over the range of Knudsen numbers and velocities tested, we found that $\gamma=1.5$, gives good results, which are, however, almost identical to those produced from the bulk equations.

Figure 3 shows results for $\mathrm{Kn}=0.05$ and a velocity difference of $v_{W}^{L}-v_{W}^{0}=200 \frac{\mathrm{m}}{\mathrm{s}}$. The differences between bulk solution and Grad13 solutions are well below the noise of the DSMC data. In the bulk of the flow, i.e. outside the Knudsen layers, we observe excellent match between the DSMC data, Grad 13 equations, and bulk equations.

As the wall velocity grows, it becomes impossible to produce reasonable results from the numerical solutions. Figure 4 shows results for $\mathrm{Kn}=0.05$ and $v_{W}^{L}-v_{W}^{0}=600 \frac{\mathrm{m}}{\mathrm{s}}$. Oscillations at the boundaries disturb the flow in the bulk, and the results differ considerably from DSMC. Note that the code is stable, and converges to these results. The bulk equations, on the other hand, provide an excellent fit to DSMC data outside the boundary layers.

As the Knudsen number grows, the breakdown of the results appears at lower velocities. At lower velocities the numerical G13 solution agrees well with the bulk solution. Since the Knudsen layers are wider, the differences to DSMC are more pronounced. Figure 5 shows the results for $\mathrm{Kn}=0.1,300 \frac{\mathrm{m}}{\mathrm{s}}$. At this Knudsen number we could not obtain reasonable results for higher velocities.

The bulk equations agree surprisingly well with DSMC even for higher velocities. As an example, Fig. 6 compares DSMC and bulk solution for $\mathrm{Kn}=0.1, v_{W}^{L}-v_{W}^{0}=600 \frac{\mathrm{m}}{\mathrm{s}}$. The figure also shows results obtained from the NSF equations with first order boundary conditions. We observe a strikingly good agreement between bulk solution and DSMC. For this case, due to the large shear rate, nonlinear contributions are more important than the linear Knudsen layers, and thus the heat flux parallel to the wall, $q_{1}$, is well described by the bulk solution. Note that for NSF $q_{1}=\sigma_{22}=0$ (these curves are not shown in the figure). We also point out that the bulk solution matches the DSMC temperature maximum in the middle of the channel very well, while the NSF equations yields markedly lower temperatures. This difference in temperatures is mostly due to the term $\frac{1}{4} V^{2}$ in the second order jump condition $(27)_{2}$ which does not appear in the boundary condition (28) that was used for the numerical solution of NSF.

At higher Knudsen numbers, the bulk solution agree less with the DSMC data, even in the bulk. The reason for this becomes clear when one remembers that the Grad13 equations are equations of second order in the Knudsen number [15]. As the Knudsen number grows, higher order effects-Knudsen layers and higher order non-linear contributions - affect the results. Figure 7 shows a comparison of DSMC, bulk equations and NSF. While the bulk equations agree better with DSMC than NSF, they clearly differ. 

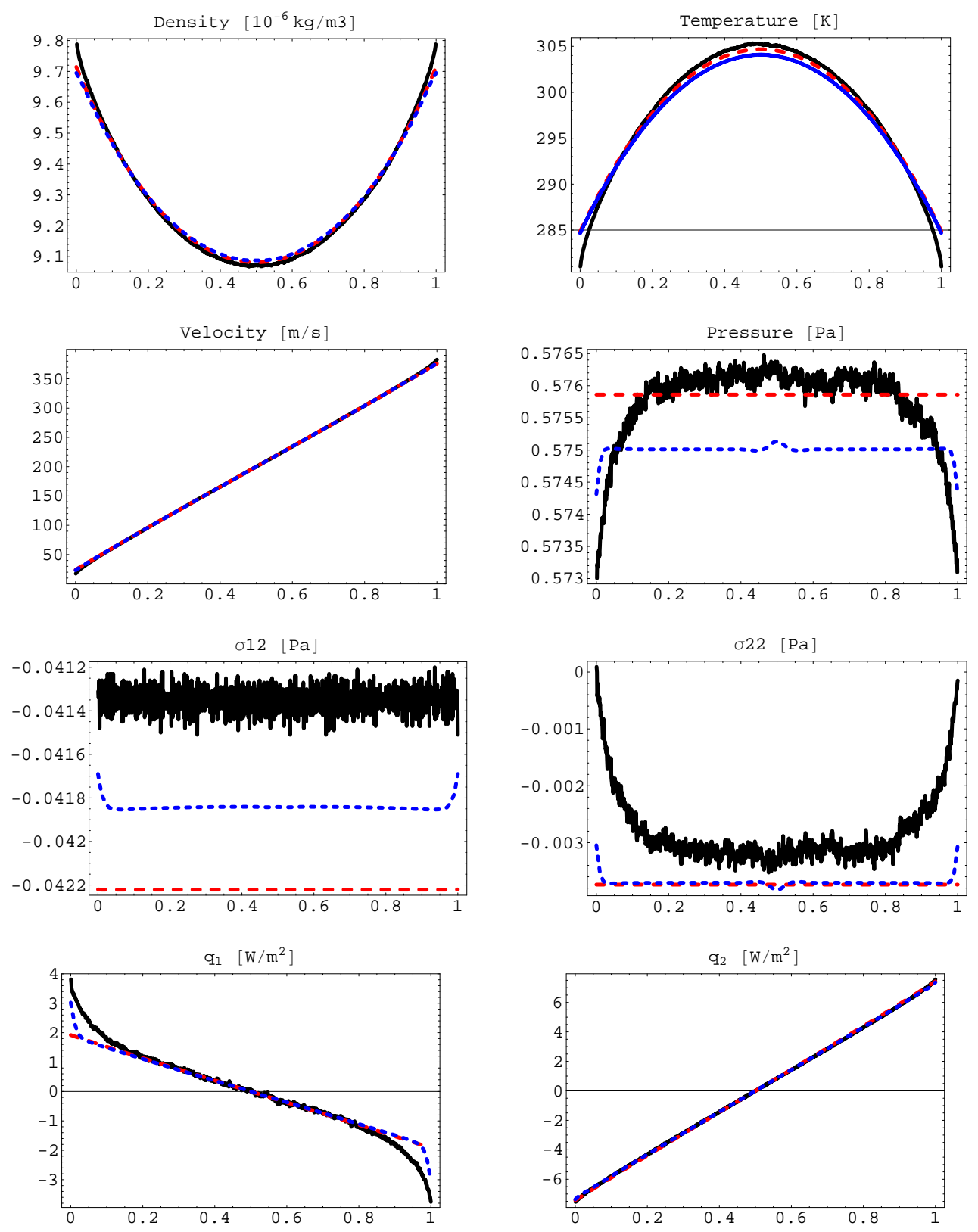

Figure 2: Couette flow results for $\mathrm{Kn}=0.05, v_{W}^{L}-v_{W}^{0}=400 \frac{\mathrm{m}}{\mathrm{s}}$ for $\gamma=1.3$. Numerical solution of G13 equations (short dashes/blue), Bulk solution (long dashes/red) and DSMC solution (continuous/black).

\section{Conclusions}

We have considered Grad's 13 moment equations for planar Couette flow. The equations require not only the usual jump and slip boundary conditions (to second order) for temperature and velocity, but also boundary conditions for normal stress. All boundary conditions were derived from Maxwell's boundary conditions for the Boltzmann equation, based on Grad's 13 moment distribution function.

Numerical solutions show good agreement to DSMC solutions for Knudsen numbers 0.1 and smaller. For larger Knudsen numbers, and for larger velocities, the numeri- cal solutions show unphysical oscillations near to the wall which lead to wrong results in the bulk. Whenever the numerical solutions are good, they closely match solutions of the bulk equations, which were derived from the Grad 13 equations in Couette geometry by considering only terms up to second order in the Knudsen number. The bulk solutions require only jump and slip conditions for temperature and velocity, and are easy to solve.

Neither set of equations can describe Knudsen boundary layers, and thus one cannot expect agreement with DSMC at larger Knudsen numbers where Knudsen layers extend far into the channel (see Fig. 7 for $\mathrm{Kn}=0.25$ ). For $\mathrm{Kn}=$ 

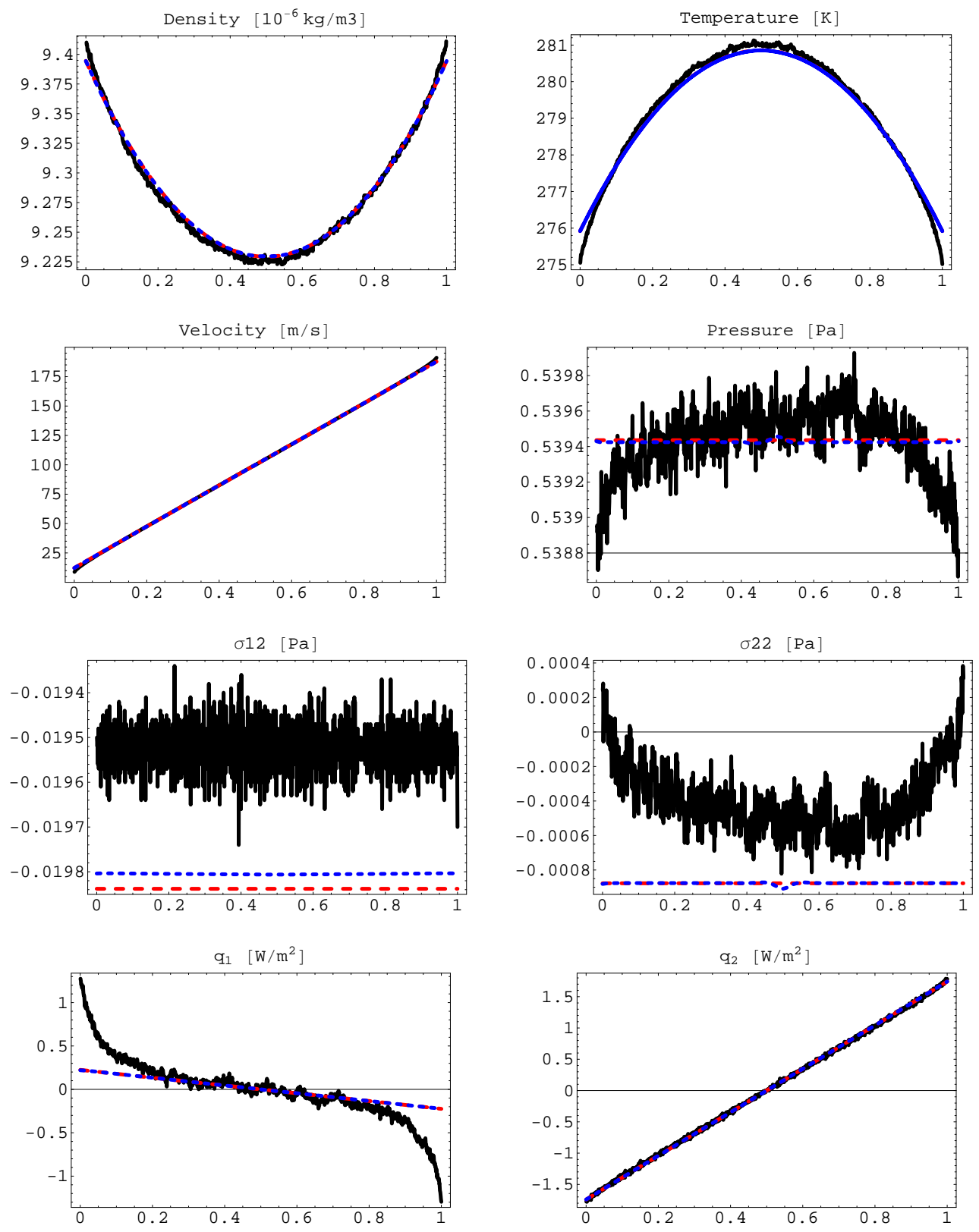

Figure 3: Couette flow results for $\mathrm{Kn}=0.05, v_{W}^{L}-v_{W}^{0}=200 \frac{\mathrm{m}}{\mathrm{s}}$ for $\gamma=1.5$. Numerical solution of G13 equations (short dashes/blue), Bulk solution (long dashes/red) and DSMC solution (continuous/black).

0.1 and less, the Grad 13 equations and the bulk equations give good results. If one is not interested in a detailed description of the Knudsen layer, the Grad 13 equations could be a useful tool for the description of flows at these Knudsen numbers.

Interestingly, our results for the G13 equations yield a constant pressure (at least outside the non-linear transition layer). This gives some support to the a priori assumption of constant pressure in Ref. [18]. Our results agree essentially with those presented in Ref. [18]. The main difference is that we solve the full boundary value numerically for given values of wall velocities and temperatures, while the analytical solution in Ref. [18] needs the shear rate as input, and then deduces the corresponding boundary values for temperature and velocity. The results of Ref. [19] cannot be compared in detail, since those authors assume a constant relaxation time $\tau=\mu / p$, and ignore jump and slip.

The new boundary condition for normal stress works well for smaller Knudsen numbers and velocities. The unphysical solutions at higher Knudsen numbers and velocities are not well understood. They seem to be due to the strong non-linear character of the equations. It might be that small inaccuracies in the boundary conditions are 

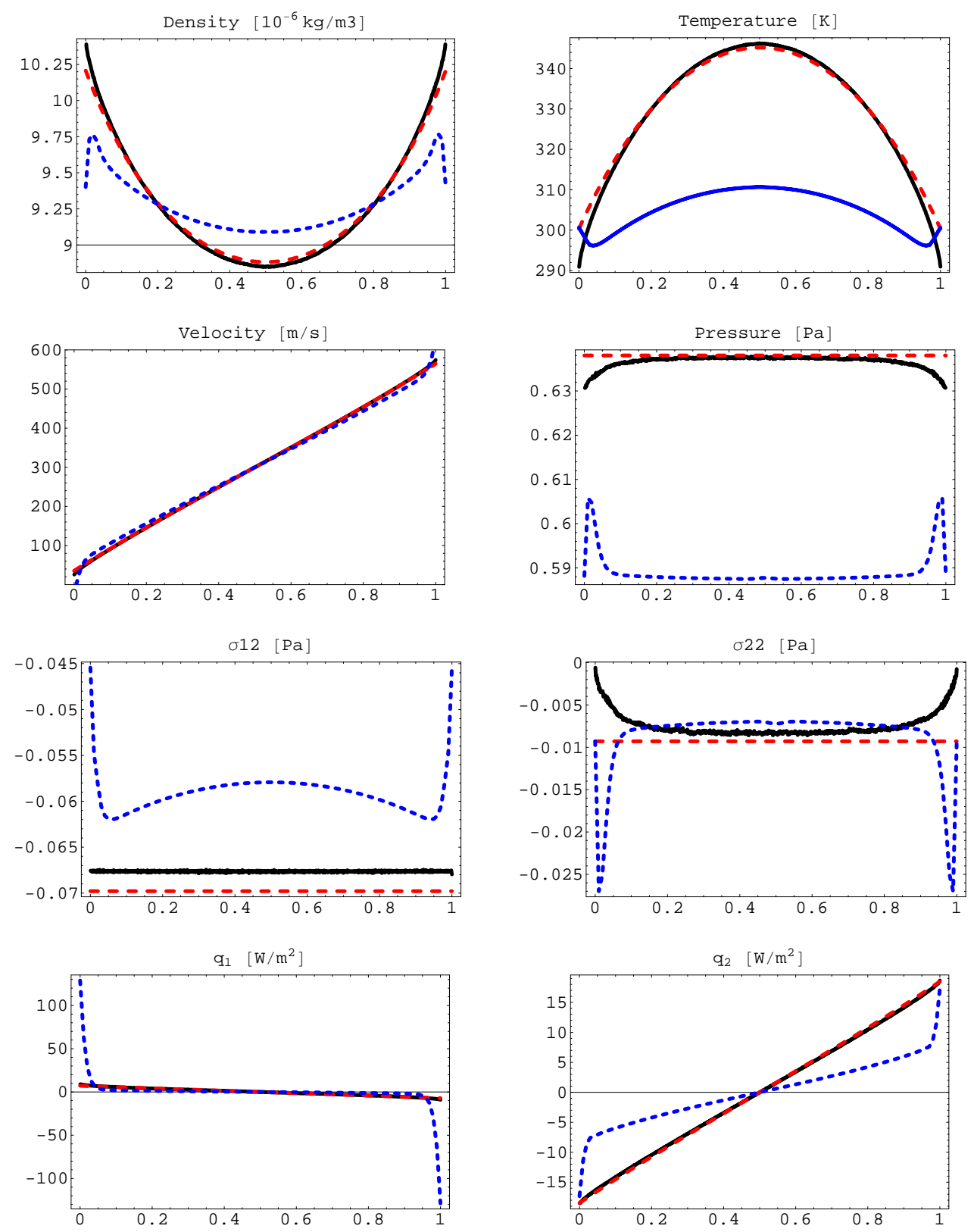

Figure 4: Couette flow results for $\mathrm{Kn}=0.05, v_{W}^{L}-v_{W}^{0}=600 \frac{\mathrm{m}}{\mathrm{s}}$ for $\gamma=1.5$. Numerical solution of G13 equations (short dashes/blue), Bulk solution (long dashes/red) and DSMC solution (continuous/black).

amplified to give results like those in Fig. 4.

Further research is necessary to better understand boundary conditions for higher order equations for rarefied gases. We see the presented results as an encouraging first step, and will aim in the future to provide a full set of boundary conditions for the regularized 13 moment equations (R13) which are accurate to third order in the Knudsen number.

Acknowledgement: This research was supported by the Natural Sciences and Engineering Research Council (NSERC). We are pleased to acknowledge helpful discussions with Giovanni Russo (Catania) on the UCS2 scheme.

\section{A Grad 13 equations in conservative form}

In conservative form the equations for $\sigma_{11}$ and $v_{2}$ are not uncoupled form the other equations and thus cannot be neglected. The conservative form of the Grad 13 equations in dimensionless form for Couette flow are [17]

$$
\begin{aligned}
\frac{\partial \rho}{\partial t}+\frac{\partial}{\partial y}\left(\rho v_{2}\right) & =0, \\
\frac{\partial \rho v_{1}}{\partial t}+\frac{\partial}{\partial y}\left(\sigma_{12}+\rho v_{1} v_{2}\right) & =0, \\
\frac{\partial \rho v_{2}}{\partial t}+\frac{\partial}{\partial y}\left(\sigma_{22}+p+\rho v_{2}^{2}\right) & =0,
\end{aligned}
$$



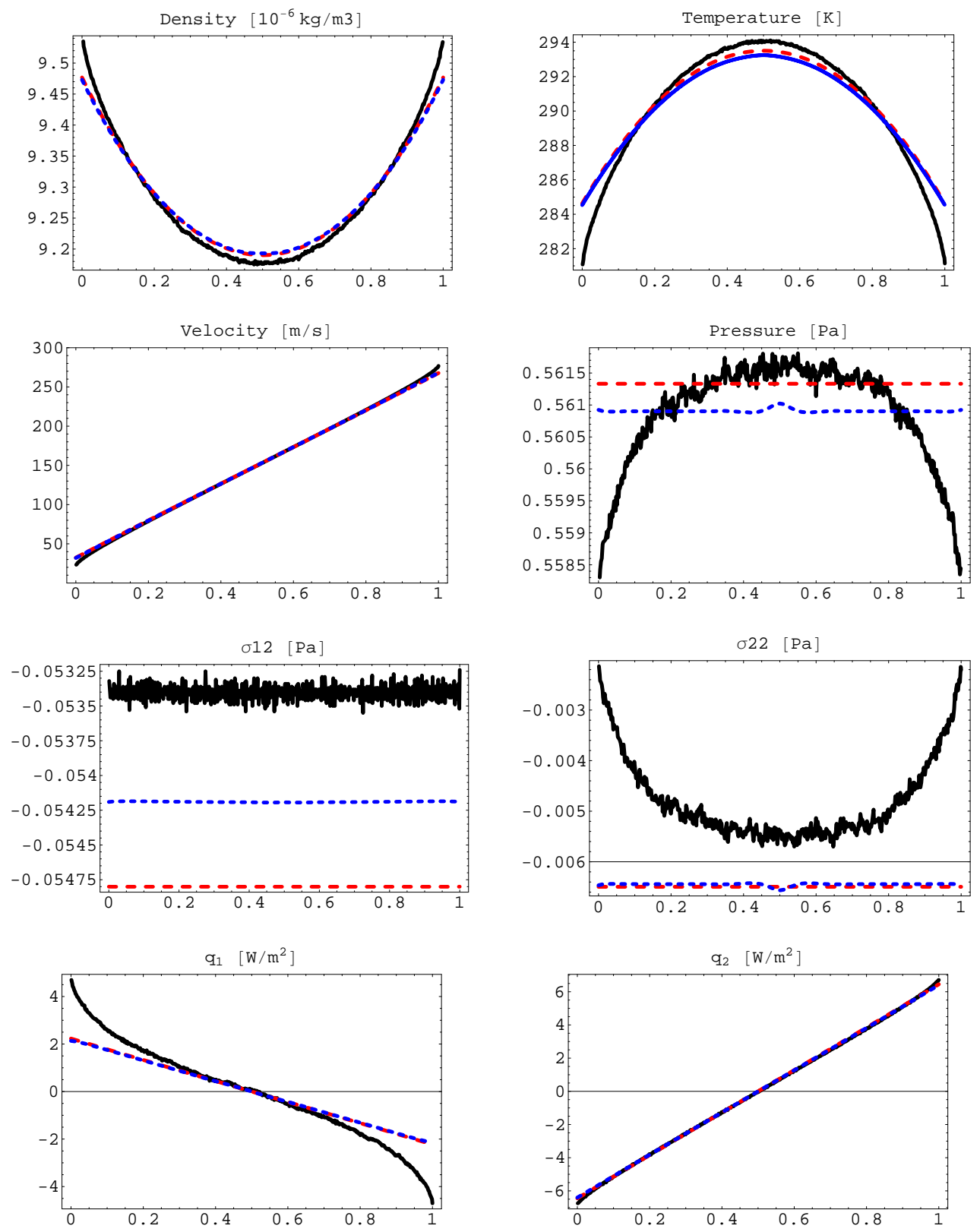

Figure 5: Couette flow results for $\mathrm{Kn}=0.1, v_{W}^{L}-v_{W}^{0}=300 \frac{\mathrm{m}}{\mathrm{s}}$ for $\gamma=1.5$. Numerical solution of G13 equations (short dashes/blue), Bulk solution (long dashes/red) and DSMC solution (continuous/black).

$\frac{\partial}{\partial t}\left(\frac{3}{2} p+\frac{\rho}{2}\left(v_{1}^{2}+v_{2}^{2}\right)\right)$

$+\frac{\partial}{\partial y}\left(\frac{5}{2} p v_{2}+q_{2}+\sigma_{12} v_{1}+\sigma_{22} v_{2}+\frac{\rho}{2}\left(v_{1}^{2}+v_{2}^{2}\right) v_{2}\right)=0$

$\frac{\partial}{\partial t}\left(\sigma_{12}+\rho v_{1} v_{2}\right)$

$+\frac{\partial}{\partial y}\left(\frac{2}{5} q_{1}+v_{1}\left(\sigma_{22}+p\right)+2 v_{2} \sigma_{12}+\rho v_{1} v_{2} v_{2}\right)=-\frac{p}{\mu} \frac{\sigma_{12}}{\mathrm{Kn}}$, $\frac{\partial}{\partial t}\left(p+\sigma_{11}+\rho v_{1}^{2}\right)$

$+\frac{\partial}{\partial y}\left(\frac{2}{5} q_{2}+2 v_{1} \sigma_{12}+v_{2}\left(\sigma_{11}+p\right)+\rho v_{1}^{2} v_{2}\right)=-\frac{p}{\mu} \frac{\sigma_{11}}{\mathrm{Kn}}$,

$$
\begin{aligned}
\frac{\partial}{\partial t}(p & \left.+\sigma_{22}+\rho v_{2}^{2}\right) \\
& +\frac{\partial}{\partial y}\left(\frac{6}{5} q_{2}+3 v_{2}\left(\sigma_{22}+p\right)+\rho v_{2}^{2} v_{2}\right)=-\frac{p}{\mu} \frac{\sigma_{22}}{\mathrm{Kn}}
\end{aligned}
$$



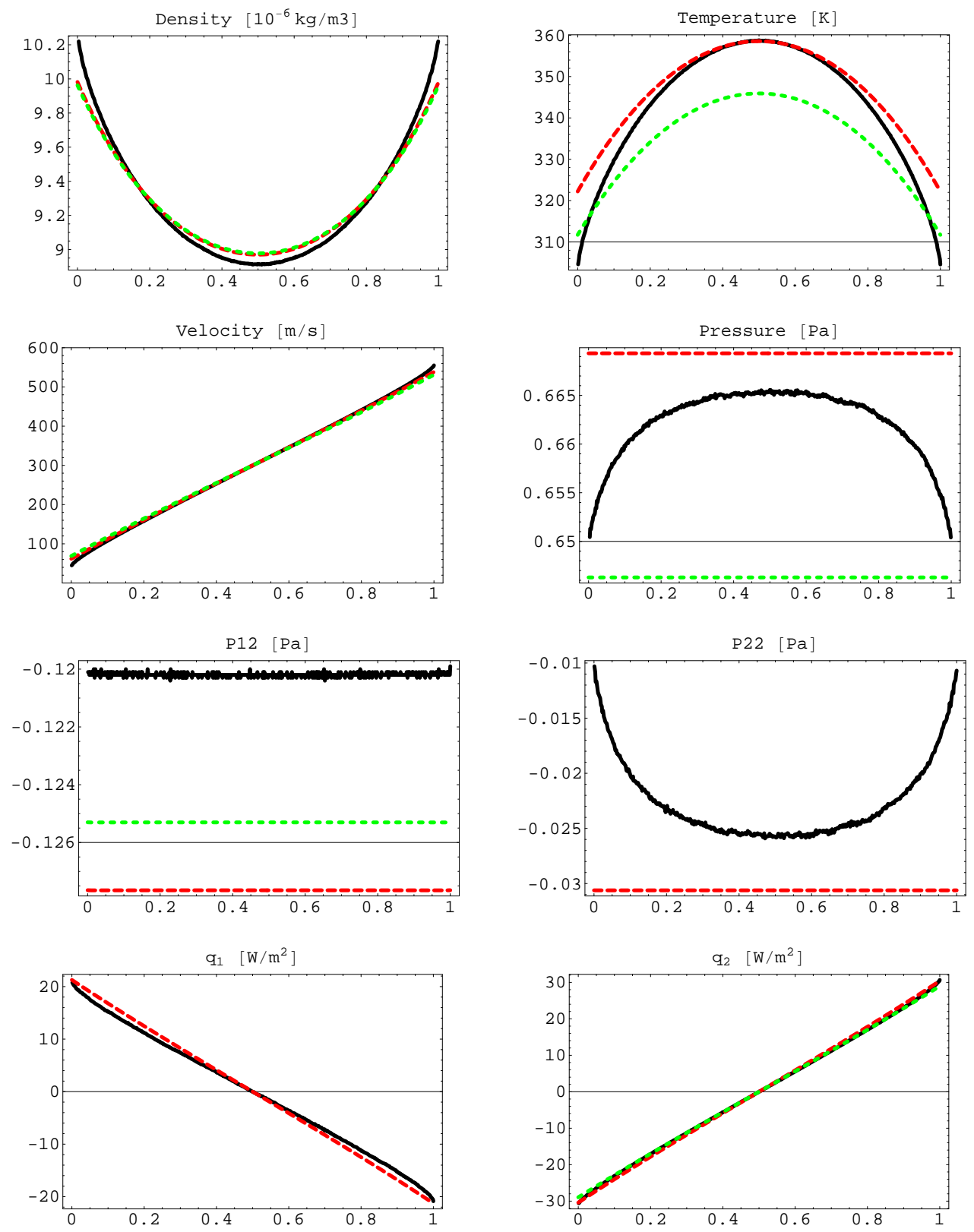

Figure 6: Couette flow results for $\mathrm{Kn}=0.1, v_{W}^{L}-v_{W}^{0}=600 \frac{\mathrm{m}}{\mathrm{s}}$. Bulk solution (long dashes/red), NSF solution (short dashes/green) and DSMC solution (continuous/black).

$$
\begin{array}{rr}
\frac{\partial}{\partial t}\left(q_{1}+\sigma_{11} v_{1}+\sigma_{12} v_{2}+\frac{5}{2} p v_{1}+\frac{1}{2} \rho\left(v_{1}^{2}+v_{2}^{2}\right) v_{1}\right) & \frac{\partial}{\partial t}\left(q_{2}+\sigma_{22} v_{2}+\sigma_{12} v_{1}+\frac{5}{2} p v_{2}+\frac{1}{2} \rho\left(v_{1}^{2}+v_{2}^{2}\right) v_{2}\right) \\
+\frac{\partial}{\partial y}\left(\frac{7}{2} \frac{p}{\rho} \sigma_{12}+\frac{7}{5} q_{1} v_{2}+\frac{7}{5} q_{2} v_{1}+\frac{1}{2} v^{2} \sigma_{12}+v_{1}^{2} \sigma_{12}\right. & +\frac{\partial}{\partial y}\left(\frac{7}{2} \frac{p}{\rho} \sigma_{22}+\frac{5}{2} \frac{p^{2}}{\rho}+\frac{16}{5} v_{2} q_{2}+\frac{2}{5} q_{1} v_{1}\right. \\
\left.+v_{2} v_{1} \sigma_{22}+v_{2}^{2} \sigma_{12}+v_{1} v_{2} \sigma_{11}+\frac{7}{2} v_{1} v_{2} p+\frac{1}{2} \rho v^{2} v_{1} v_{2}\right) & \left.+\frac{1}{2} v^{2}\left(p+\sigma_{22}\right)+2 v_{1} v_{2} \sigma_{12}+2 v_{2}^{2} \sigma_{22}+\frac{7}{2} v_{2}^{2} p+\frac{1}{2} \rho v^{2} v_{2}^{2}\right) \\
=-\frac{p}{\mu} \frac{1}{\mathrm{Kn}}\left(v_{1} \sigma_{11}+v_{2} \sigma_{12}+\frac{2}{3} q_{1}\right), & =-\frac{p}{\mu} \frac{1}{\mathrm{Kn}}\left(v_{1} \sigma_{12}+v_{2} \sigma_{22}+\frac{2}{3} q_{2}\right) .
\end{array}
$$



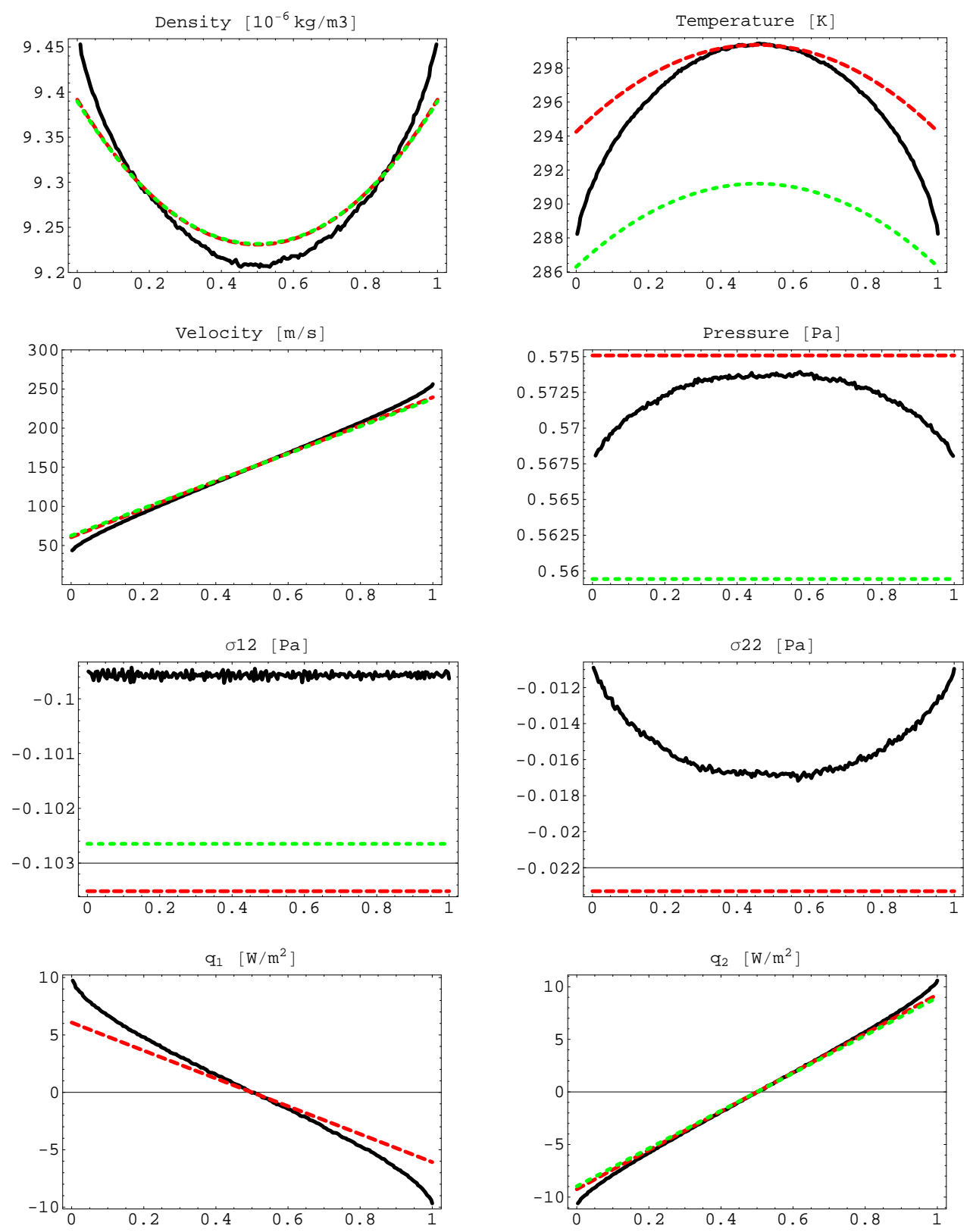

Figure 7: Couette flow results for Kn $=0.25, v_{W}^{L}-v_{W}^{0}=300 \frac{m}{s}$. Bulk solution (long dashes/red), NSF solution (short dashes/green) and DSMC solution (continuous/black).

\section{REFERENCES}

[1] C. Cercignani (1975) Theory and application of the Boltzmann Equation. Scottish Academic Press, Edinburgh

[2] M.N. Kogan (1969) Rarefied Gas Dynamics. Plenum Press, New York

[3] S. Chapman and T. G. Cowling (1970) The mathematical Theory of Non-Uniform Gases. Cambridge University Press
[4] H. Struchtrup (2005) Macroscopic Transport Equations for Rarefied Gas Flows-Approximation Methods in Kinetic Theory. Interaction of Mechanics and Mathematics Series, Springer, Heidelberg

[5] A.V. Bobylev (1982) 'The Chapman-Enskog and Grad methods for solving the Boltzmann equation'. Sov. Phys. Dokl. Vol.27, pp.29-31.

[6] P. Rosenau (1989) 'Extending hydrodynamics via the regularization of the Chapman-Enskog solution', Phys. Rev. A Vol.40, pp.7193-7196. 
[7] H. Grad (1949) 'On the Kinetic Theory of Rarefied Gases', Comm. Pure Appl. Math. Vol.2, pp.325.

[8] H. Grad (1958) Principles of the Kinetic Theory of Gases. in Handbuch der Physik XII: Thermodynamik der Gase, S. Flügge (Ed.), Springer, Berlin

[9] Zhong, X., MacCormack, R. W., and Chapman, D. R. (1993) 'Stabilization of the Burnett Equations and Applications to Hypersonic Flows', AIAA Journal Vol.31, pp.1036.

[10] S. Jin and M. Slemrod (2001) 'Regularization of the Burnett equations via relaxation', J. Stat. Phys. Vol.103, pp.1009-1033.

[11] A.V. Bobylev (2006), 'Instabilities in the ChapmanEnskog Expansion and Hyperbolic Burnett Equations', J. Stat. Phys., Vol.124, pp.371-399.

[12] I. Müller, D. Reitebuch, and W. Weiss (2003) 'Extended Thermodynamics - Consistent in Order of Magnitude', Cont. Mech. Thermodyn. Vol.15, pp.113146.

[13] Struchtrup, H. and Torrilhon, M. (2003) 'Regularization of Grad's 13-moment-equations: Derivation and Linear Analysis', Phys. Fluids Vol.15, pp.2668-2680.

[14] M. Torrilhon and H. Struchtrup (2004) 'Regularized 13-Moment-Equations: Shock Structure Calculations and Comparison to Burnett Models', J. Fluid Mech. Vol.513, pp.171-198.

[15] H. Struchtrup (2004) 'Stable transport equations for rarefied gases at high orders in the Knudsen number', Phys. Fluids Vol.16, pp.3921-3934.

[16] H. Struchtrup (2004) 'Derivation of 13 moment equations for rarefied gas flow to second order accuracy for arbitrary interaction potentials', Multiscale Model. Simul. Vol.3, pp.211-243.

[17] T. Thatcher, M.A.Sc. thesis, University of Victoria, 2005.

[18] W. Marques Jr. and G.M. Kremer (2001) 'Couette flow from a thirteen field theory with jump and slip boundary conditions', Continuum Mech. Thermodyn. Vol.13, pp.207-217.

[19] I.-S. Liu and M.A. Rincon (2004) 'A boundary value problem in extended thermodynamics: one dimensional steady flows with heat conduction', Continuum Mech. Thermodyn. Vol.16, pp.109-124.

[20] R.E. Street (1961) 'Plane Couette Flow by the Methods of Moments', Proc. Rarefied Gas Dynamics, Ed. L Talbot, Academic Press, New York.

[21] S. Kubota and Y. Yoshizawa (1974) 'Couette flow problems by thirteen moment equations', Proc. Rarefied Gas Dynamics, M. Becker and M. Fiebig (Eds.), DFVLR Press, Porz-Wahn.
[22] H. Struchtrup and T. Thatcher (2006) 'Bulk equations and Knudsen layers for the regularized 13 moment equations', submitted.

[23] D.A. Lockerby and J.M. Reese (2003) 'High resolution Burnett simulation of micro Couette flow and heat transfer', J. Comp. Phys. Vol.188, pp.333-347.

[24] H. Struchtrup and W. Weiss (2000) 'Temperature jumps and velocity slip in the moment method', Cont. Mech. Thermodyn. Vol.12, pp.1-18.

[25] T. Ohwada, Y. Sone, and K. Aoki (1989) 'Numerical analysis of the shear and thermal creep flows of a rarefied gas over a plane wall on the basis of the linearized Boltzmann equation for hard-sphere molecules', Phys. Fluids A Vol.1(9), pp.1588-1599.

[26] F. Sharipov and V. Seleznev (1998) 'Data on Internal Rarefied Gas Flows', J. Phys.Chem. Ref. Data Vol.27(3), pp.657-706.

[27] F. Sharipov (2003) 'Application of the CercignaniLampis scattering kernel to calculations of rarefied gas flows. II. Slip and jump coefficients', European J. of Mechanics - B/Fluids Vol.22(2), pp.133-143.

[28] Y. Sone, Kinetic Theory and Fluid dynamics. Birkhäuser, Boston 2002.

[29] D.A. Lockerby, J.M. Reese, D.R. Emerson, and R.W. Barber (2004) 'Velocity boundary condition at solid walls in rarefied gas calculations', Phys. Rev. E Vol.70, 017303.

[30] H. Struchtrup (2005) 'Failures of the Burnett and Super-Burnett equations in steady state processes', Cont. Mech. Thermodyn. Vol.17, pp.43-50.

[31] S.F. Liotta, V. Romano, and G. Russo (2000) 'Central Schemes for Balance Laws of Relaxation Type, SIAM J. Num. Analysis Vol.38(4), pp.1337-1356.

[32] H. Nessyahu and E. Tadmor (1990) 'Non-oscillatory Central Differencing for Hyperbolic Conservation Laws', J. Comp. Phys. Vol.87(2), pp.408-463. 